\title{
A HYBRID MEAN VALUE OF THE INVERSION OF $L$-FUNCTIONS AND GENERAL QUADRATIC GAUSS SUMS*
}

\author{
WENPENG ZHANG AND YUPING DENG
}

\begin{abstract}
The main purpose of this paper is, using the estimates for character sums and the analytic method, to study the $2 k$-th power mean of the inversion of Dirichlet $L$-functions with the weight of general quadratic Gauss sums, and give two interesting asymptotic formulas.
\end{abstract}

\section{$\S 1$. Introduction}

Let $q \geq 2$ be an integer, $\chi$ denotes a Dirichlet character modulo $q$. For any integer $n$, we define the general quadratic Gauss sums $G(n, \chi ; q)$ as follows:

$$
G(n, \chi ; q)=\sum_{a=1}^{q} \chi(a) e\left(\frac{n a^{2}}{q}\right)
$$

where $e(y)=e^{2 \pi i y}$. This sum is important, because it is a generalization of the classical Gauss sums and quadratic Gauss sums. But about the properties of $G(n, \chi ; q)$, we know very little at present, we do not know even how large $|G(n, \chi ; q)|$ is. The main purpose of this paper is, using the estimates for character sums and the analytic method, to study the asymptotic distribution of the $2 k$-th power mean

$$
\sum_{p \leq Q} \frac{1}{p \cdot(p-1)^{2}} \sum_{\chi \bmod p} \frac{|G(n, \chi ; p)|^{4}}{|L(1, \chi)|^{2 k}},
$$

and give a sharp asymptotic formula, where $Q$ is any positive number with $Q \geq 2, p$ denotes an odd prime, $L(s, \chi)$ denotes the Dirichlet $L$-function corresponding to character $\chi \bmod p$. In fact, we shall prove the following:

Received April 28, 2000.

2000 Mathematics Subject Classification: 11M20.

*This work is supported by the N.S.F. and the P.N.S.F. of P.R.China. 
TheOREM 1. Let $p$ denote an odd prime with $p \leq Q$. Then for any fixed positive integer $k$ and integer $n$ with $|n| \leq Q$, we have the asymptotic formula

$$
\sum_{p \leq Q} \frac{1}{p(p-1)^{2}} \sum_{\chi \bmod p} \frac{|G(n, \chi ; p)|^{4}}{|L(1, \chi)|^{2 k}}=3 \cdot C(k) \cdot \pi(Q)+O\left(Q^{\frac{1}{2}+\epsilon}\right),
$$

where $C(k)=\prod_{p}\left[1+\frac{\left(C_{k}^{1}\right)^{2}}{p^{2}}+\frac{\left(C_{k}^{2}\right)^{2}}{p^{4}}+\cdots+\frac{\left(C_{k}^{k}\right)^{2}}{p^{2 k}}\right], \sum_{\chi \bmod p}$ denotes the summation over all characters modulo $p, \prod_{p}$ denotes the product over all primes, $\pi(Q)$ denotes the number of all primes $p$ satisfying $2 \leq p \leq Q, \epsilon$ denotes any fixed positive number, and $C_{k}^{i}=\frac{k !}{(k-i) ! i !}$.

THEOREM 2. Let $Q \geq 3$ be a real number. Then for any fixed positive integer $k$ and any integer $n$ with $|n| \leq Q$, we have the asymptotic formula

$$
\sum_{p \leq Q}^{\prime} \frac{1}{p^{2}(p-1)^{2}} \sum_{\chi \bmod p} \frac{|G(n, \chi ; p)|^{6}}{|L(1, \chi)|^{2 k}}=10 \cdot C(k) \cdot \pi_{1}(Q)+O\left(Q^{\frac{1}{2}+\epsilon}\right),
$$

where $\sum^{\prime}$ denotes the summation over all primes $p$ with $p \leq Q$ and $p \equiv$ $3 \bmod 4, \pi_{1}(Q)$ denotes the number of all primes $p$ satisfying $2 \leq p \leq Q$ and $p \equiv 3 \bmod 4$.

\section{$\S 2$. Some lemmas}

In order to complete the proof of the theorems, we need following several lemmas.

Lemma 1. Let $y \geq 2$ be a real number, $k$ be any positive integer. Then for

$$
r(n)=\sum_{n_{1} n_{2} \cdots n_{k}=n} \mu\left(n_{1}\right) \cdots \mu\left(n_{k}\right)
$$

we have an estimate

$$
\sum_{q \leq Q} \sup _{\substack{a, x \\ x \leq y}}\left|\sum_{\substack{n \leq x \\ n \equiv a(q)}} r(n)\right| \ll_{A, k} y(\ln y)^{-A}+y^{1-\frac{1}{2^{k}}} Q(\ln (y Q))^{4},
$$

where $\mu(n)$ is the Möbius function, $A$ is any positive number, $\ll_{A, k}$ denote the constants implied by the symbols $\ll$ depend only on parameter $A$ and $k$. 
Proof. (See reference [5]).

Lemma 2. Let $p$ denote an odd prime with $p \leq Q, \chi$ denote a Dirichlet character modulo $p$. Then for any positive integer $k$, we have an estimate

$$
\sum_{p \leq Q} \frac{1}{p^{\frac{3}{2}}} \sum_{a=1}^{p-1}\left|\sum_{\chi \bmod p} \frac{\chi(a)}{|L(1, \chi)|^{2 k}}\right|=O\left(Q^{\frac{1}{2}+\epsilon}\right),
$$

where $\epsilon$ denotes any fixed positive number.

Proof. For convenience, firstly we write

$$
A(\chi, y)=\sum_{\frac{p}{a}<n \leq y} \chi(n) r(n), \quad B(\chi, y)=\sum_{p<n \leq y} \chi(n) r(n)
$$

where $a$ is any positive integer with $1 \leq a<p$. If $\operatorname{Re}(s)>1$ and $\chi \neq \chi_{0}$ (principal character $\bmod p$ ), then we have

$$
\frac{1}{L^{k}(s, \chi)}=\sum_{n=1}^{\infty} \frac{\chi(n) r(n)}{n^{s}}
$$

From (3) and Abel's identity we have

$$
\frac{1}{L^{k}(s, \chi)}=\sum_{n \leq N} \frac{\chi(n) r(n)}{n^{s}}+s \int_{N}^{+\infty} \frac{A(\chi, y)}{y^{s+1}} d y
$$

By the property of Dirichlet $L$-function and $L(1, \chi) \neq 0$ we know that $(4)$ is also correct if $s=1$. So we have

$$
\begin{aligned}
\frac{1}{L^{k}(1, \chi)} & =\sum_{1 \leq n<\frac{p}{a}} \frac{\chi(n) r(n)}{n}+\int_{\frac{p}{a}}^{+\infty} \frac{A(\chi, y)}{y^{2}} d y \\
& =\sum_{1 \leq n<p} \frac{\chi(n) r(n)}{n}+\int_{p}^{+\infty} \frac{B(\chi, y)}{y^{2}} d y
\end{aligned}
$$

Hence from $(3),(5)$ and note that $\frac{1}{L\left(1, \chi_{0}\right)}=0$ we get

$$
\sum_{\chi \bmod p} \frac{\chi(a)}{|L(1, \chi)|^{2 k}}=\sum_{\chi \bmod p}^{\prime} \chi(a)\left|\sum_{n=1}^{\infty} \frac{\chi(n) r(n)}{n}\right|^{2}
$$




$$
\begin{aligned}
& =\sum_{\chi \bmod p}^{\prime} \chi(a)\left(\sum_{1 \leq n<\frac{p}{a}} \frac{\chi(n) r(n)}{n}+\int_{\frac{p}{a}}^{+\infty} \frac{A(\chi, y)}{y^{2}} d y\right) \\
& \times\left(\sum_{1 \leq m<p} \frac{\bar{\chi}(m) r(m)}{m}+\int_{p}^{+\infty} \frac{B(\bar{\chi}, y)}{y^{2}} d y\right) \\
& =\sum_{\chi \bmod p}^{\prime} \chi(a)\left(\sum_{1 \leq n<\frac{p}{a}} \frac{\chi(n) r(n)}{n}\right)\left(\sum_{1 \leq m<p} \frac{\bar{\chi}(m) r(m)}{m}\right) \\
& +\sum_{\chi \bmod p}^{\prime} \chi(a)\left(\sum_{1 \leq n<\frac{p}{a}} \frac{\chi(n) r(n)}{n}\right)\left(\int_{p}^{+\infty} \frac{B(\bar{\chi}, y)}{y^{2}} d y\right) \\
& +\sum_{\chi \bmod p}^{\prime} \chi(a)\left(\sum_{1 \leq m<p} \frac{\bar{\chi}(m) r(m)}{m}\right)\left(\int_{\frac{p}{a}}^{+\infty} \frac{A(\chi, y)}{y^{2}} d y\right) \\
& +\sum_{\chi \bmod p}^{\prime} \chi(a)\left(\int_{\frac{p}{a}}^{+\infty} \frac{A(\chi, y)}{y^{2}} d y\right)\left(\int_{p}^{+\infty} \frac{B(\bar{\chi}, y)}{y^{2}} d y\right) \\
& \left.\equiv M_{1}+M_{3}+M_{4}, \quad \sum_{1}\right)
\end{aligned}
$$

where $\sum_{\chi \bmod p}^{\prime}$ denotes the summation over all nonprincipal characters mod $p$. So from the above we have

$$
\begin{aligned}
& \sum_{p \leq Q} \frac{1}{p^{\frac{3}{2}}} \sum_{a=1}^{p-1}\left|\sum_{\chi \bmod p} \frac{\chi(a)}{|L(1, \chi)|^{2 k}}\right| \\
& \quad \leq \sum_{p \leq Q} \frac{1}{p^{\frac{3}{2}}} \sum_{a=1}^{p-1}\left(\left|M_{1}\right|+\left|M_{2}\right|+\left|M_{3}\right|+\left|M_{4}\right|\right)
\end{aligned}
$$

Now we shall estimate each term in expression (6). From the orthogonality relation for characters modulo $p$ we know that for $(p, m n)=1$,

$$
\sum_{\chi \bmod p} \chi(n) \bar{\chi}(m)= \begin{cases}p-1, & \text { if } n \equiv m \bmod p \\ 0, & \text { otherwise. }\end{cases}
$$

From this identity we can easily get

(7) $M_{1}=\sum_{\chi \bmod p} \chi(a)\left(\sum_{1 \leq n<\frac{p}{a}} \frac{\chi(n) r(n)}{n}\right)\left(\sum_{1 \leq m<p} \frac{\bar{\chi}(m) r(m)}{m}\right)+O\left(p^{\epsilon}\right)$ 


$$
\begin{aligned}
& =(p-1) \sum_{\substack { 1 \leq n<\frac{p}{a} \\
\begin{subarray}{c}{1 \leq m<p \\
a n \equiv m(p){ 1 \leq n < \frac { p } { a } \\
\begin{subarray} { c } { 1 \leq m < p \\
a n \equiv m ( p ) } }\end{subarray}} \frac{r(n) r(m)}{n m}+O\left(p^{\epsilon}\right) \\
& =(p-1) \sum_{1 \leq n<\frac{p}{a}} \frac{r(n) r(a n)}{a n^{2}}+O\left(p^{\epsilon}\right) \\
& \leq \frac{p r(a)}{a} \sum_{1 \leq n<\frac{p}{a}} \frac{|r(n)|^{2}}{n^{2}}+O\left(p^{\epsilon}\right) \\
& \leq \frac{p r(a)}{a} \sum_{\substack{n=1 \\
(n, p)=1}}^{\infty} \frac{|r(n)|^{2}}{n^{2}}+O\left(p^{\epsilon}\right) .
\end{aligned}
$$

Note that

$$
\sum_{\substack{n=1 \\(n, p)=1}}^{\infty} \frac{r^{2}(n)}{n^{2}}=\prod_{\substack{p_{1} \\ p_{1} \neq p}}\left[1+\frac{\left(C_{k}^{1}\right)^{2}}{p_{1}^{2}}+\frac{\left(C_{k}^{2}\right)^{2}}{p_{1}^{4}} \cdots+\frac{\left(C_{k}^{k}\right)^{2}}{p_{1}^{2 k}}\right] \ll C,
$$

an absolute constant, and the estimate $r(n) \leq d_{k}(n) \ll p^{\epsilon}$, from (7) we immediately get

$$
\sum_{p \leq Q} \frac{1}{p^{\frac{3}{2}}} \sum_{a=1}^{p-1}\left|M_{1}\right| \ll \sum_{p \leq Q} \frac{1}{p^{\frac{1}{2}}} \sum_{a=1}^{p-1} \frac{r(a)}{a}+\sum_{p \leq Q} \frac{1}{p^{\frac{3}{2}}} \sum_{a=1}^{p-1} p^{\epsilon} \ll Q^{\frac{1}{2}+\epsilon} .
$$

(9) $\sum_{p \leq Q} \frac{1}{p^{\frac{3}{2}}} \sum_{a=1}^{p-1}\left|M_{2}\right|$

$$
\begin{aligned}
& =\sum_{p \leq Q} \frac{1}{p^{\frac{3}{2}}} \sum_{a=1}^{p-1}\left|\sum_{\chi \bmod p}^{\prime} \chi(a)\left(\sum_{n<\frac{p}{a}} \frac{\chi(n) r(n)}{n}\right)\left(\int_{p}^{+\infty} \frac{B(\bar{\chi}, y)}{y^{2}} d y\right)\right| \\
& \leq \sum_{p \leq Q} \frac{1}{p^{\frac{3}{2}}} \sum_{a=1}^{p-1}\left|\sum_{\chi \bmod p}^{\prime} \chi(a)\left(\sum_{n<\frac{p}{a}} \frac{\chi(n) r(n)}{n}\right)\left(\int_{p}^{Q^{2^{k}}} \frac{B(\bar{\chi}, y)}{y^{2}} d y\right)\right| \\
& \quad+\sum_{p \leq Q} \frac{1}{p^{\frac{3}{2}}} \sum_{a=1}^{p-1}\left|\sum_{\chi \bmod p}^{\prime} \chi(a)\left(\sum_{n<\frac{p}{a}} \frac{\chi(n) r(n)}{n}\right)\left(\int_{Q^{2^{k}}}^{+\infty} \frac{B(\bar{\chi}, y)}{y^{2}} d y\right)\right| \\
& \leq \sum_{p \leq Q} \frac{1}{p^{\frac{3}{2}}} \sum_{a=1}^{p-1} \mid \sum_{\chi \bmod p} \chi(a)\left(\sum_{n<\frac{p}{a}} \frac{\chi(n) r(n)}{n}\right)\left(\int_{p}^{Q^{2^{k}}} \frac{\left.\sum_{p<m \leq y} \frac{\bar{\chi}(m) r(m)}{y^{2}} d y\right) \mid}{}\right.
\end{aligned}
$$




$$
\begin{aligned}
& +\sum_{p \leq Q} \frac{1}{p^{\frac{1}{2}}} \sum_{a=1}^{p-1} \sum_{n<\frac{p}{a}} \frac{|r(n)|}{n}\left|\int_{Q^{2^{k}}}^{+\infty} \frac{1}{y^{2}}\left(\sum_{\substack{p<m \leq y \\
m \equiv n a(p)}} r(m)\right) d y\right|+O\left(Q^{\frac{1}{2}+\epsilon}\right) \\
& \leq \sum_{p \leq Q} \frac{1}{p^{\frac{3}{2}}} \sum_{a=1}^{p-1}\left|\sum_{\chi \bmod p} \chi(a)\left(\sum_{n<\frac{p}{a}} \frac{\chi(n) r(n)}{n}\right)\left(\int_{p}^{Q^{2^{k}}} \frac{\sum_{p<m \leq y} \bar{\chi}(m) r(m)}{y^{2}} d y\right)\right| \\
& +O\left(Q^{\frac{1}{2}+\epsilon} \int_{Q^{2^{k}}}^{+\infty} \frac{1}{y^{2}} \sum_{p \leq Q} \sup _{\substack{a, z \\
z \leq y}}\left|\sum_{\substack{m \leq z \\
m \equiv a(p)}} r(m)\right| d y\right)+O\left(Q^{\frac{1}{2}+\epsilon}\right) .
\end{aligned}
$$

So from Lemma 1 (taking $A=2$ ) and (9) we have

$$
\begin{aligned}
& \sum_{p \leq Q} \frac{1}{p^{\frac{3}{2}}} \sum_{a=1}^{p-1}\left|M_{2}\right| \\
\leq & \sum_{p \leq Q} \frac{1}{p^{\frac{1}{2}}} \int_{p}^{Q^{2^{k}}} \frac{1}{y^{2}}\left[\sum_{a=1}^{p-1} \sum_{n<\frac{p}{a}} \sum_{\substack{p<m \leq y \\
a n \equiv m(p)}} \frac{r(n) r(m)}{n}\right] d y+O\left(Q^{\frac{1}{2}+\epsilon}\right) \\
& +O\left(Q^{\frac{1}{2}+\epsilon} \int_{Q^{2^{k}}}^{+\infty} \frac{1}{y^{2}}\left[y(\ln y)^{-A}+y^{1-\frac{1}{2^{k}}} Q(\ln (y Q))^{4}\right] d y\right) \\
= & \left(\sum_{p \leq Q} \frac{1}{p^{\frac{1}{2}}} \sum_{a=1}^{p-1} \sum_{n<\frac{p}{a}} \frac{1}{n} \int_{p}^{Q^{2^{k}}} \frac{y \cdot \frac{1}{p} \cdot p^{\epsilon}}{y^{2}} d y\right) \\
& +O\left(Q^{\frac{1}{2}+\epsilon} \int_{Q^{2^{k}}}^{+\infty}\left[y^{-1}(\ln y)^{-A}+y^{-1-\frac{1}{2^{k}}} Q(\ln (y Q))^{4}\right] d y\right) \\
= & \left(\sum_{p \leq Q} p^{-\frac{1}{2}+\epsilon}\right)_{+} O\left(Q^{\frac{1}{2}+\epsilon}\right) \\
= & \left(Q^{\frac{1}{2}+\epsilon}\right) \cdot
\end{aligned}
$$

Applying the same method of proving (10), we also have the estimate

$$
\begin{aligned}
& \sum_{p \leq Q} \frac{1}{p^{\frac{3}{2}}} \sum_{a=1}^{p-1}\left|M_{3}\right| \\
= & \sum_{p \leq Q} \frac{1}{p^{\frac{3}{2}}} \sum_{a=1}^{p-1}\left|\sum_{\chi \bmod p}^{\prime} \chi(a)\left(\sum_{m \leq p} \frac{\bar{\chi}(m) r(m)}{m}\right)\left(\int_{\frac{p}{a}}^{+\infty} \frac{A(\chi, y)}{y^{2}} d y\right)\right|
\end{aligned}
$$




$$
\begin{aligned}
& \leq \sum_{p \leq Q} \frac{1}{p^{\frac{3}{2}}} \sum_{a=1}^{p-1}\left|\sum_{\chi \bmod p}^{\prime} \chi(a)\left(\sum_{m \leq p} \frac{\bar{\chi}(m) r(m)}{m}\right)\left(\int_{\frac{p}{a}}^{Q^{2^{k}}} \frac{A(\chi, y)}{y^{2}} d y\right)\right| \\
& +\sum_{p \leq Q} \frac{1}{p^{\frac{3}{2}}} \sum_{a=1}^{p-1}\left|\sum_{\chi \bmod p}^{\prime} \chi(a)\left(\sum_{m \leq p} \frac{\bar{\chi}(m) r(m)}{m}\right)\left(\int_{Q^{2^{k}}}^{+\infty} \frac{A(\chi, y)}{y^{2}} d y\right)\right| \\
& \leq \sum_{p \leq Q} \frac{1}{p^{\frac{3}{2}}} \sum_{a=1}^{p-1}\left|\sum_{\chi \bmod p} \chi(a)\left(\sum_{m<p} \frac{\bar{\chi}(m) r(m)}{m}\right)\left(\int_{\frac{p}{a}}^{Q^{2^{k}}} \frac{\sum_{\frac{p}{a}<n \leq y} \chi(n) r(n)}{y^{2}} d y\right)\right| \\
& +\sum_{p \leq Q} \frac{1}{p^{\frac{1}{2}}} \sum_{a=1}^{p-1} \int_{Q^{2} k}^{+\infty} \frac{1}{y^{2}}\left|\sum_{m<p} \frac{r(m)}{m} \sum_{\substack{p \\
a \\
a n \equiv m \leq y}} r(n)\right| d y+O\left(Q^{\frac{1}{2}+\epsilon}\right)
\end{aligned}
$$

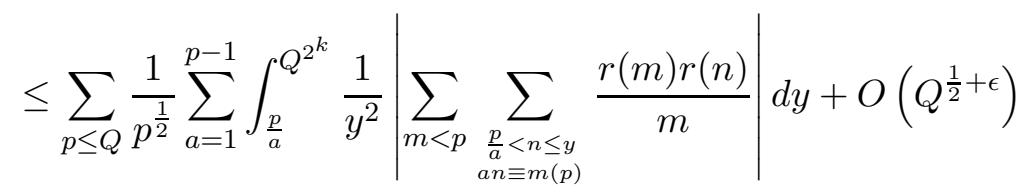

$$
\begin{aligned}
& +O\left(Q^{\frac{1}{2}+\epsilon} \int_{Q^{2^{k}}}^{+\infty} \frac{1}{y^{2}} \sum_{p \leq Q} \sup _{\substack{a, z \\
z \leq y}}\left|\sum_{\substack{n \leq z \\
n \equiv a(p)}} r(n)\right| d y\right) \\
& =O\left(\sum_{p \leq Q} \frac{p}{p^{\frac{1}{2}}} \sum_{m<p} \frac{1}{m} \int_{\frac{p}{a}}^{Q^{2^{k}}} \frac{y \cdot \frac{1}{p} \cdot p^{\epsilon}}{y^{2}} d y\right)+O\left(Q^{\frac{1}{2}+\epsilon}\right) \\
& =O\left(Q^{\frac{1}{2}+\epsilon}\right) \text {. }
\end{aligned}
$$

where we have used the estimate $r(n) \ll n^{\epsilon}$ and for any fixed positive integers $h$ and $m$, the number of the solutions of equation $a n=h p+m$ (for $a$ and $n)$ is $\ll d(h p+m) \ll p^{\epsilon}$. Similarly, we can also get the estimate

$$
\sum_{p \leq Q} \frac{1}{p^{\frac{3}{2}}} \sum_{a=1}^{p-1}\left|\sum_{\chi \bmod p}^{\prime} \chi(a) \int_{\frac{p}{a}}^{+\infty} \frac{A(\chi, y)}{y^{2}} d y \int_{p}^{+\infty} \frac{B(\bar{\chi}, z)}{z^{2}} d z\right|=O\left(Q^{\frac{1}{2}+\epsilon}\right)
$$

Combining (6), (8), (10), (11) and (12) we obtain the estimate

$$
\sum_{p \leq Q} \frac{1}{p^{\frac{3}{2}}} \sum_{a=1}^{p-1}\left|\sum_{\chi \bmod p} \frac{\chi(a)}{\mid L(1, \chi)^{2 k}}\right|=O\left(Q^{\frac{1}{2}+\epsilon}\right) .
$$

This proves Lemma 2. 
LEMMA 3. Let $Q \geq 2$, then for any fixed integer $k$, we have the asymptotic formula

$$
\sum_{p \leq Q} \frac{1}{\phi(p)} \sum_{\substack{\chi \neq \chi_{0} \\ \chi(-1)=1}} \frac{1}{|L(1, \chi)|^{2 k}}=\frac{1}{2} \cdot C(k) \cdot \pi(Q)+O\left(Q^{\frac{1}{2}+\epsilon}\right) .
$$

Proof. For prime power $p^{\alpha}$, note that $\mu(1)=1, \mu(p)=-1$ and $\mu\left(p^{i}\right)=$ 0 if $i>1$. So that we have the identity

$$
r\left(p^{\alpha}\right)=\sum_{n_{1} n_{2} \cdots n_{k}=p^{\alpha}} \mu\left(n_{1}\right) \mu\left(n_{2}\right) \cdots \mu\left(n_{k}\right)= \begin{cases}(-1)^{\alpha} C_{k}^{\alpha}, & \text { if } \alpha \leq k \\ 0, & \text { otherwise }\end{cases}
$$

and the asymptotic formula

$$
\begin{aligned}
\sum_{\substack{n=1 \\
(n, p)=1}}^{\infty} \frac{r^{2}(n)}{n^{2}} & =\prod_{\substack{p_{1} \\
p_{1} \neq p}}\left[1+\frac{\left(C_{k}^{1}\right)^{2}}{p_{1}^{2}}+\frac{\left(C_{k}^{2}\right)^{2}}{p_{1}^{4}}+\cdots+\frac{\left(C_{k}^{k}\right)^{2}}{p_{1}^{2 k}}\right] \\
& =C(k)+O\left(\frac{1}{p^{2}}\right),
\end{aligned}
$$

from the orthogonality relation for characters and the method of proving Lemma 2 we can easily get

$$
\begin{aligned}
& \sum_{\substack{\chi \neq \chi_{0} \\
\chi(-1)=1}}\left|\sum_{n \leq Q^{2^{k}}} \frac{r(n) \chi(n)}{n}\right|^{2} \\
= & \frac{\phi(p)}{2} \sum_{\substack{n \leq Q^{2^{k}} \\
(n, p)=1}} \frac{r^{2}(n)}{n^{2}}+\frac{\phi(p)}{2} \sum_{\substack { n \leq Q^{2^{k}} \\
\begin{subarray}{c}{m \leq Q^{2^{k}} \\
m \equiv \pm n(p) \\
m \neq n{ n \leq Q ^ { 2 ^ { k } } \\
\begin{subarray} { c } { m \leq Q ^ { 2 ^ { k } } \\
m \equiv \pm n ( p ) \\
m \neq n } }\end{subarray}} \frac{r(n) r(m)}{n m}+O\left(Q^{\epsilon}\right) \\
= & \frac{\phi(p)}{2} \cdot C(k)+O\left(Q^{\epsilon}\right), \\
& \sum_{p \leq Q} \frac{1}{\phi(p)} \sum_{\substack{\chi \neq \chi_{0} \\
\chi(-1)=1}}\left(\sum_{n \leq Q^{2^{k}}} \frac{r(n) \bar{\chi}(n)}{n}\right) \int_{Q^{2^{k}}}^{\infty} \frac{A(y, \chi)}{y^{2}} d y \ll Q^{\frac{1}{2}+\epsilon}
\end{aligned}
$$

and

$$
\sum_{p \leq Q} \frac{1}{\phi(p)} \sum_{\substack{\chi \neq \chi_{0} \\ \chi(-1)=1}}\left|\int_{Q^{2^{k}}}^{\infty} \frac{A(y, \chi)}{y^{2}} d y\right|^{2} \ll Q^{\frac{1}{2}+\epsilon}
$$


where $A(y, \chi)=\sum_{Q^{2^{k}}<n \leq y} r(n) \chi(n)$. Note that the identity

$$
\frac{1}{L^{k}(1, \chi)}=\sum_{n=1}^{+\infty} \frac{r(n) \chi(n)}{n}=\sum_{n \leq Q^{2^{k}}} \frac{r(n) \chi(n)}{n}+\int_{Q^{2^{k}}}^{\infty} \frac{A(y, \chi)}{y^{2}} d y
$$

Combining the above asymptotic formula and the estimates we have

$$
\begin{aligned}
& \sum_{p \leq Q} \frac{1}{\phi(p)} \sum_{\substack{\chi \neq \chi_{0} \\
\chi(-1)=1}} \frac{1}{|L(1, \chi)|^{2 k}} \\
& =\sum_{p \leq Q} \frac{1}{\phi(p)} \sum_{\substack{\chi \neq \chi_{0} \\
\chi(-1)=1}}\left|\sum_{n \leq Q^{2} k} \frac{r(n) \chi(n)}{n}\right|^{2} \\
& +\sum_{p \leq Q} \frac{1}{\phi(p)} \sum_{\substack{\chi \neq \chi_{0} \\
\chi(-1)=1}}\left(\sum_{n \leq Q^{2^{k}}} \frac{r(n) \chi(n)}{n}\right) \int_{Q^{2^{k}}}^{\infty} \frac{A(y, \bar{\chi})}{y^{2}} d y \\
& +\sum_{p \leq Q} \frac{1}{\phi(p)} \sum_{\substack{\chi \neq \chi_{0} \\
\chi(-1)=1}}\left(\sum_{n \leq Q^{2 k}} \frac{r(n) \bar{\chi}(n)}{n}\right) \int_{Q^{2^{k}}}^{\infty} \frac{A(y, \chi)}{y^{2}} d y \\
& +\sum_{p \leq Q} \frac{1}{\phi(p)} \sum_{\substack{\chi \neq \chi_{0} \\
\chi(-1)=1}}\left|\int_{Q^{2^{k}}}^{\infty} \frac{A(y, \chi)}{y^{2}} d y\right|^{2} \\
& =\frac{1}{2} \cdot C(k) \cdot \pi(Q)+O\left(Q^{\frac{1}{2}+\epsilon}\right) .
\end{aligned}
$$

This proves Lemma 3.

LEMMA 4. For any integer $q \geq 1$, we have the calculation formula

$$
G(1 ; q)=\frac{1}{2} \sqrt{q}(1+i)\left(1+e^{\frac{-\pi i q}{2}}\right)= \begin{cases}\sqrt{q} & \text { if } q \equiv 1(\bmod 4) \\ 0 & \text { if } q \equiv 2(\bmod 4) \\ i \sqrt{q} & \text { if } q \equiv 3(\bmod 4) \\ (1+i) \sqrt{q} & \text { if } q \equiv 0(\bmod 4)\end{cases}
$$

where $G(1 ; q)=\sum_{a=1}^{q} e\left(\frac{a^{2}}{q}\right)$ 
Proof. This is a remarkable formula of Gauss. See the formula (30) of $[1]$.

LEMMA 5. Let $p$ be an odd prime, $\chi$ be any nonprincipal even character (i.e. $\chi(-1)=1$ and $\left.\chi \neq \chi_{0}\right) \bmod p$. Then for any integer $n$ with $(n, p)=1$, we have the identity

$$
|G(n, \chi ; p)|^{2}=2 p+\left(\frac{n}{p}\right) G(1 ; p) \sum_{a=1}^{p-1} \chi(a)\left(\frac{a^{2}-1}{p}\right),
$$

where $\left(\frac{n}{p}\right)$ is the Legendre symbol.

Proof. First we let $G(n ; p)=\sum_{a=1}^{p} e\left(\frac{n a^{2}}{p}\right)$. Then for $p \nmid n$, from the formula (29) of [1] we know that

$$
G(n ; p)=\left(\frac{n}{p}\right) G(1 ; p) .
$$

Applying (13) we can get that if $\chi$ is a nonprincipal even character $\bmod p$, then

$$
\begin{aligned}
|G(n, \chi ; p)|^{2} & =\sum_{a=1}^{p-1} \sum_{b=1}^{p-1} \chi(a) \bar{\chi}(b) e\left(\frac{n a^{2}-n b^{2}}{p}\right) \\
& =\sum_{a=1}^{p-1} \sum_{b=1}^{p-1} \chi(a \bar{b}) e\left(\frac{n\left(a^{2}-b^{2}\right)}{p}\right) \\
& =\sum_{a=1}^{p-1} \sum_{b=1}^{p-1} \chi(a) e\left(\frac{n b^{2}\left(a^{2}-1\right)}{p}\right) \\
& =\sum_{a=1}^{p-1} \chi(a)\left[\sum_{b=1}^{p} e\left(\frac{n b^{2}\left(a^{2}-1\right)}{p}\right)-1\right] \\
& =\sum_{a=1}^{p-1} \chi(a) G\left(n\left(a^{2}-1\right) ; p\right)-\sum_{a=1}^{p-1} \chi(a) \\
& =2 G(0 ; p)+\sum_{a=2}^{p-2} \chi(a) G\left(n\left(a^{2}-1\right) ; p\right) \\
& =2 p+\left(\frac{n}{p}\right) G(1 ; p) \sum_{a=1}^{p-1} \chi(a)\left(\frac{a^{2}-1}{p}\right)
\end{aligned}
$$

This proves Lemma 5. 


\section{§3. Proof of the theorems}

In this section, we shall complete the proof of the theorems. First note that if $\chi$ is an odd character modulo $p$ (or $\chi \neq \chi_{0}$ and $p \mid n$ ), then

$$
G(n, \chi ; p)=\sum_{a=1}^{p} \chi(a) e\left(\frac{a^{2} n}{p}\right)=0 .
$$

So for any integer $n$, from Lemma 5 we have

$$
\begin{aligned}
& \sum_{p \leq Q} \frac{1}{p \phi^{2}(p)} \sum_{\chi \bmod p} \frac{|G(n, \chi ; p)|^{4}}{|L(1, \chi)|^{2 k}} \\
& =\sum_{\substack{p \leq Q \\
(p, n)=1}} \frac{1}{p \phi^{2}(p)} \sum_{\substack{\chi \neq \chi_{0} \\
\chi(-1)=1}} \frac{|G(n, \chi ; p)|^{4}}{|L(1, \chi)|^{2 k}} \\
& =\sum_{\substack{p \leq Q \\
(p, n)=1}} \frac{1}{p \phi^{2}(p)} \sum_{\substack{\chi \neq \chi_{0} \\
\chi(-1)=1}}\left[2 p+\left(\frac{n}{p}\right) G(1 ; p) \sum_{a=1}^{p-1} \chi(a)\left(\frac{a^{2}-1}{p}\right)\right]^{2} \\
& =\sum_{\substack{p \leq Q \\
(p, n)=1}} \frac{1}{p \phi^{2}(p)} \sum_{\substack{\chi \neq \chi_{0} \\
\chi(-1)=1}}\left[4 p^{2}+4 p\left(\frac{n}{p}\right) G(1 ; p) \sum_{a=1}^{p-1} \chi(a)\left(\frac{a^{2}-1}{p}\right)\right] \\
& +\sum_{\substack{p \leq Q \\
(p, n)=1}} \frac{G^{2}(1 ; p)}{p \phi^{2}(p)} \sum_{\substack{\chi \neq \chi_{0} \\
\chi(-1)=1}} \sum_{a=1}^{p-1} \sum_{b=1}^{p-1} \chi(a b)\left(\frac{a^{2}-1}{p}\right)\left(\frac{b^{2}-1}{p}\right) \\
& \mid L(1, \chi)^{2 k} \\
& |L(1, \chi)|^{2 k}
\end{aligned}
$$

On the other hand, we have the identities

$$
\begin{aligned}
\sum_{a=1}^{p-1} \sum_{b=1}^{p-1} \chi(a b)\left(\frac{a^{2}-1}{p}\right)\left(\frac{b^{2}-1}{p}\right) \\
=\sum_{a=1}^{p-1} \sum_{b=1}^{p-1} \chi(a)\left(\frac{a^{2} \bar{b}^{2}-1}{p}\right)\left(\frac{b^{2}-1}{p}\right)
\end{aligned}
$$




$$
\begin{gathered}
=\sum_{a=1}^{p-1} \sum_{b=1}^{p-1} \chi(a)\left(\frac{a^{2}-b^{2}}{p}\right)\left(\frac{b^{2}-1}{p}\right) \\
=2\left(\frac{-1}{p}\right)(p-3)+\sum_{a=2}^{p-2} \sum_{b=1}^{p-1} \chi(a)\left(\frac{a^{2}-b^{2}}{p}\right)\left(\frac{b^{2}-1}{p}\right) \\
\sum_{a=2}^{p-2}\left(\frac{a^{2}-b^{2}}{p}\right) \sum_{\chi(-1)=-1} \frac{\chi(a)}{\mid L(1, \chi)^{2 k}} \\
=\sum_{a=1}^{p-1}\left(\frac{a^{2}-1}{p}\right) \sum_{\chi(-1)=-1} \frac{\chi(a)}{|L(1, \chi)|^{2 k}}=0 .
\end{gathered}
$$

From the Lemma 1 of [8] we can also deduce the estimate

$$
\sum_{b=1}^{p-1}\left(\frac{b^{2}-a^{2}}{p}\right)\left(\frac{b^{2}-1}{p}\right) \ll \sqrt{p}, \quad a^{2} \not \equiv 1 \bmod p .
$$

Now combining (14), (15), (16), Lemma 2, Lemma 3 and Lemma 4 we have

$$
\begin{aligned}
& \sum_{p \leq Q} \frac{1}{p \phi^{2}(p)} \sum_{\chi \bmod p} \frac{|G(n, \chi ; p)|^{4}}{|L(1, \chi)|^{2 k}} \\
& =\sum_{\substack{p \leq Q \\
(p, n)=1}} \frac{1}{p \phi^{2}(p)}\left(4 p^{2}+G^{2}(1 ; p) \cdot 2\left(\frac{-1}{p}\right)(p-3)\right) \sum_{\substack{\chi \neq \chi_{0} \\
\chi(-1)=1}} \frac{1}{|L(1, \chi)|^{2 k}} \\
& +\sum_{\substack{p \leq Q \\
(p, n)=1}} \frac{1}{p \phi^{2}(p)} 4 p\left(\frac{n}{p}\right) G(1 ; p) \sum_{a=1}^{p-1}\left(\frac{a^{2}-1}{p}\right) \sum_{\substack{\chi \neq \chi_{0} \\
\chi(-1)=1}} \frac{\chi(a)}{|L(1, \chi)|^{2 k}} \\
& +\sum_{\substack{p \leq Q \\
(p, n)=1}} \frac{G^{2}(1 ; p)}{p \phi^{2}(p)} \sum_{a=2}^{p-2} \sum_{b=1}^{p-1}\left(\frac{a^{2}-b^{2}}{p}\right)\left(\frac{b^{2}-1}{p}\right) \sum_{\substack{\chi \neq \chi_{0} \\
\chi(-1)=1}} \frac{\chi(a)}{|L(1, \chi)|^{2 k}} \\
& =\sum_{p \leq Q} \frac{6 p^{2}-6 p}{p(p-1)^{2}} \sum_{\substack{\chi \neq \chi_{0} \\
\chi(-1)=1}} \frac{1}{|L(1, \chi)|^{2 k}}+O\left(Q^{\epsilon}\right) \\
& +O\left(\sum_{p \leq Q} \frac{1}{p^{\frac{3}{2}}} \sum_{a=2}^{p-2}\left|\sum_{\chi \neq \chi_{0}} \frac{\chi(a)}{\mid L(1, \chi)^{2 k}}\right|\right) \\
& =3 \cdot C(k) \cdot \pi(Q)+O\left(Q^{\frac{1}{2}+\epsilon}\right) \text {. }
\end{aligned}
$$


This proves Theorem 1 .

Now we prove Theorem 2. From Lemma 5 we have

$$
\begin{aligned}
& \sum_{p \leq Q}^{\prime} \frac{1}{p^{2} \phi^{2}(p)} \sum_{\chi \bmod p} \frac{|G(n, \chi ; p)|^{6}}{|L(1, \chi)|^{2 k}} \\
& =\sum_{\substack{p \leq Q \\
(p, n)=1}}^{\prime} \frac{1}{p^{2} \phi^{2}(p)} \sum_{\substack{\chi \neq \chi_{0} \\
\chi(-1)=1}} \frac{|G(n, \chi ; p)|^{6}}{|L(1, \chi)|^{2 k}} \\
& =\sum_{\substack{p \leq Q \\
(p, n)=1}}^{\prime} \sum_{\substack{\chi \neq \chi_{0} \\
\chi(-1)=1}}\left[2 p+\left(\frac{n}{p}\right) G(1 ; p) \sum_{a=1}^{p-1} \chi(a)\left(\frac{a^{2}-1}{p}\right)\right]^{3} \frac{1}{|L(1, \chi)|^{2 k}}
\end{aligned}
$$

For $(p, n)=1$ and $p \equiv 3 \bmod 4$,

$$
\begin{aligned}
& \sum_{\chi \neq \chi_{0}} \frac{|G(n, \chi ; p)|^{6}}{|L(1, \chi)|^{2 k}}=\sum_{\chi \neq \chi_{0}} \frac{|\overline{G(n, \chi ; p)}|^{6}}{|\overline{L(1, \chi)}|^{2 k}} \\
& \quad=\sum_{\chi \neq \chi_{0}}\left|\sum_{a=1}^{p-1} \bar{\chi}(a) e\left(\frac{-n a^{2}}{p}\right)\right|^{6} \cdot \frac{1}{|L(1, \bar{\chi})|^{2 k}} \\
& \quad=\sum_{\chi \neq \chi_{0}}\left|\sum_{a=1}^{p-1} \chi(a) e\left(\frac{-n a^{2}}{p}\right)\right|^{6} \cdot \frac{1}{|L(1, \chi)|^{2 k}} \\
& \quad=\sum_{\substack{\chi \neq \chi_{0} \\
\chi(-1)=1}}\left[2 p+\left(\frac{-n}{p}\right) G(1 ; p) \sum_{a=1}^{p-1} \chi(a)\left(\frac{a^{2}-1}{p}\right)\right]^{3} \cdot \frac{1}{|L(1, \chi)|^{2 k}} .
\end{aligned}
$$

Note that $\left(\frac{-1}{p}\right)=-1$, from $(15),(16),(17)$, (18), Lemma 2, Lemma 3 and Lemma 4 we obtain

$$
\begin{aligned}
\sum_{p \leq Q}^{\prime} & \frac{1}{p^{2} \phi^{2}(p)} \sum_{\chi \bmod p} \frac{|G(n, \chi ; p)|^{6}}{|L(1, \chi)|^{2 k}} \\
& =\frac{1}{2}\left[\sum_{p \leq Q}^{\prime} \frac{1}{p^{2} \phi^{2}(p)} \sum_{\chi \neq \chi_{0}} \frac{|G(n, \chi ; p)|^{6}}{|L(1, \chi)|^{2 k}}+\sum_{p \leq Q}^{\prime} \frac{1}{p^{2} \phi^{2}(p)} \sum_{\chi \neq \chi_{0}} \frac{|\overline{G(n, \chi ; p)}|^{6}}{|\overline{L(1, \chi)}|^{2 k}}\right]
\end{aligned}
$$




$$
\begin{aligned}
& =\sum_{\substack{p \leq Q \\
(p, n)=1}}^{\prime} \frac{1}{p^{2} \phi^{2}(p)} \sum_{\substack{\chi \neq \chi_{0} \\
\chi(-1)=1}}\left[(2 p)^{3}+6 p G^{2}(1 ; p)\left(\sum_{a=1}^{p-1} \chi(a)\left(\frac{a^{2}-1}{p}\right)\right)^{2}\right] \\
& =10 \cdot C(k) \cdot \pi_{1}(Q)+O\left(Q^{\frac{1}{2}+\epsilon}\right) .
\end{aligned}
$$

This completes the proof of Theorem 2 .

Note. For general integer $m \geq 3$, whether there exists an asymptotic formula for

$$
\sum_{p \leq Q} \frac{1}{p^{m-1}(p-1)^{2}} \sum_{\chi \bmod p} \frac{|G(n, \chi ; p)|^{2 m}}{|L(1, \chi)|^{2 k}}
$$

is an unsolved problem.

Acknowledgements. The authors express their gratitude to the referee for his very helpful and detailed comments.

\section{REFERENCES}

[1] Tom M. Apostol, Introduction to Analytic Number Theory, Springer-Verlag, New York, 1976.

[2] S. Chowla, On Kloostermann's sum, Norkse Vid. Selbsk. Fak. Frondheim, 40 (1967), 70-72.

[3] A.V. Malyshev, A generalization of Kloostermann sums and their estimates (in Russian), Vestnik Leningrad Univ., 15 (1960), 59-75.

[4] T. Estermann, On Kloostermann's sum, Mathematica, 8 (1961), 83-86.

[5] Zhang Wenpeng, On the 2k-th power mean of inversion of Dirichlet L-function, Chinese J. Contemp. Math., 14 (1993), 1-7.

[6] R.C. Vaughan, An elementary method in prime number theory, Recent Progress in Analytic Number Theory, 1 (1981), Academic Press, 341-347.

[7] H. Davenport, Multiplicative number theory, Markham, 1967.

[8] D.A. Burgess, On character sums and L-series, Proc. London Math. Soc., 12 (1962), 193-206.

Wenpeng Zhang

Department of Mathematics

Northwest University

$X i$ 'an, Shaanxi

P.R.China

wpzhang@nwu.edu.cn 
Yuping Deng

Department of Mathematics

Northwest University

$X i$ 'an, Shaanxi

P.R.China 